\title{
Identification of a New Chemotype of Anti-Obesity Compounds by Ensemble Screening
}

\author{
Hyunkyung Cho, ${ }^{\dagger}$, Joo-Youn Lee, ${ }^{\dagger, t, \xi}$ Sang Yoon Choi, ${ }^{\dagger, \xi}$ Chaemin Lim, ${ }^{\dagger}$ Min-Kyoung Park, ${ }^{\dagger}$ \\ Hyejin An, Jeong OkLee,,$^{\dagger}$ Minsoo Noh,,$^{\dagger}$ Seunghee Lee, ${ }^{*}{ }^{\dagger}$ and Sanghee Kim ${ }^{*}{ }^{\dagger}$
}

${ }^{\dagger}$ College of Pharmacy, Seoul National University, 1 Gwanak-ro, Gwanak-gu, Seoul 08826, Korea

\$Chemical Data-Driven Research Center, Korea Research Institute of Chemical Technology, 141 Gajeong-ro, Yuseong-gu, Daejeon 34114, Korea

†Korea Food Research Institute, 245 Nongsaengmyeong-ro, Iseo-myeon, Wanju-gun, Jeollabuk-do 55365, Korea

\section{Table of Contents}

I. Natural Piper-Amide-Like Library Screening Data $\ldots \ldots \ldots \ldots \ldots \ldots \ldots \ldots \ldots \ldots \ldots . . . . . . .62$

II. The ROC and enrichment plots of the Bayesian classification model $\ldots . . . . . . . . . . .53$

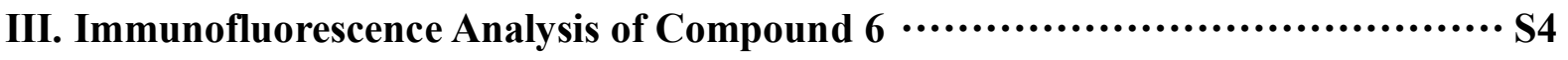

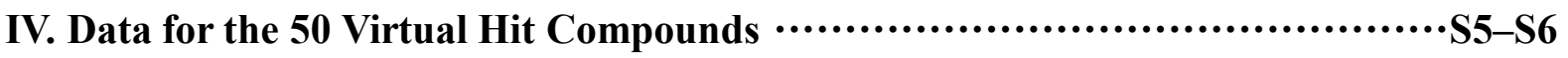

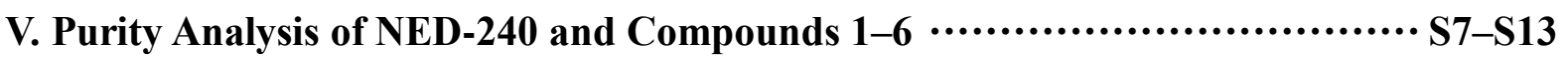

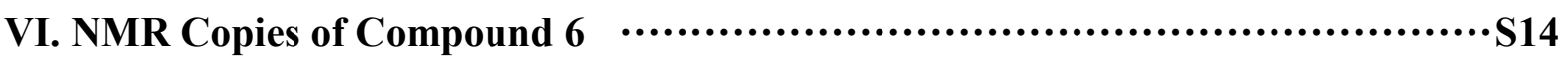

${ }^{\S}$ These authors contributed equally to this work. 


\section{Natural Piper-Amide-Like Library Screening Data}

\section{Table S1. Piper-Amide-Like Library Screening Data ${ }^{a}$}

\begin{tabular}{|c|c|c|}
\hline $\begin{array}{l}\text { Lipid inhibitory } \\
\text { activity range (\%) }\end{array}$ & Compounds & $\begin{array}{l}\text { Number of } \\
\text { compounds }\end{array}$ \\
\hline $90-$ & $\begin{array}{l}\text { NED-262, NED-278, NED-275, NED-240, NED-242, NED-223, } \\
\text { NED-225, SY-086, NED-237, NED-272, NED-241, NED-109 }\end{array}$ & 12 \\
\hline $70-90$ & $\begin{array}{l}\text { NED-180, NED-073, NED-386, NED-210, NED-261, NED-105, } \\
\text { NED-229, NED-138, NED-139, NED-134 }\end{array}$ & 10 \\
\hline $50-70$ & NED-219, SA-001, NED-384, NED-152, NED-184, NED-265 & 6 \\
\hline $30-50$ & $\begin{array}{l}\text { NED-024, NED-207, NED-216, NED-096, NED-017, NED-020, } \\
\text { NED-215, NED-199, NED-197, NED-198, NED-016 }\end{array}$ & 11 \\
\hline $10-30$ & $\begin{array}{llll}\text { NED-178, NED-030, NED-137, NED-042, } & \text { NED-385, NED-206, } \\
\text { NED-080, NED-070, NED-260, NED-048, NED-025, NED-200, } \\
\text { NED-092, SA-165, NED-230, NED-040, NED-072, NED-263, } \\
\text { NED-194, NED-193, NED-164, NED-186, NED-094, NED-204, } \\
\text { NED-203, NED-190, NED-189, NED-259, NED-037, NED-085, } \\
\text { NED-060 }\end{array}$ & 31 \\
\hline$-10-10$ & $\begin{array}{llll}\text { NED-110, NED-157, } & \text { NED-274, NED-087, } & \text { NED-062, NED-086, } \\
\text { NED-114, NED-174, NED-115, NED-177, NED-176, NED-116, } \\
\text { NED-254, NED-258, NED-130, NED-163, NED-162, NED-051, } \\
\text { NED-012, NED-009, NED-038, NED-091, NED-267, NED-171, } \\
\text { NED-288, NED-056, NED-140, NED-188, NED-057, NED-018, } \\
\text { NED-156, NED-031, NED-145, NED-147, NED-027, NED-084, } \\
\text { NED-029, NED-228, NED-149, NED-008, NED-078, NED-253, } \\
\text { NED-136, NED-158 }\end{array}$ & 44 \\
\hline$-30--10$ & $\begin{array}{l}\text { NED-113, NED-021, NED-026, NED-095, NED-192, NED-300, } \\
\text { NED-227, NED-002, NED-155, NED-047, NED-208, NED-217, } \\
\text { NED-129, NED-232, NED-076, NED-257, NED-243, NED-251, } \\
\text { NED-119, NED-043, NED-093, NED-224, SA-145, NED-154, } \\
\text { NED-273, NED-165, NED-120, NED-170, NED-003, NED-055, } \\
\text { NED-066, NED-058, NED-063, NED-023, NED-284, NED-256, } \\
\text { NED-074, NED-246, NED-112, NED-011, NED-061, NED-088, } \\
\text { NED-167, NED-054, NED-005, NED-160, NED-283, NED-035, } \\
\text { NED-231 }\end{array}$ & 49 \\
\hline$-50--30$ & $\begin{array}{l}\text { NED-006, NED-001, NED-090, NED-173, NED-079, NED-102, } \\
\text { NED-271, NED-146, NED-064, NED-266, NED-068, NED-044, } \\
\text { NED-104, NED-014, SA-054, NED-252, NED-033, NED-244, } \\
\text { NED-117, NED-081, NED-161, NED-082, NED-172, NED-124, } \\
\text { NED-183, NED-065, NED-097, NED-041, NED-067, NED-159, } \\
\text { NED-106, NED-075, NED-108, NED-191 }\end{array}$ & 34 \\
\hline--50 & $\begin{array}{lllll}\text { NED-121, NED-032, } & \text { NED-010, NED-013, NED-250, NED-201, } \\
\text { NED-127, NED-045, NED-071, NED-077, NED-028, NED-151, } \\
\text { NED-083, NED-205, NED-099, NED-202, NED-166, NED-004, } \\
\text { NED-007, NED-019, NED-153, NED-107, NED-143, NED-269, } \\
\text { NED-015, NED-069, NED-175, NED-268, NED-036, NED-034, } \\
\text { NED-144 }\end{array}$ & 31 \\
\hline
\end{tabular}

${ }^{a}$ All 228 compounds were tested on 3T3-L1 cells at $50 \mu \mathrm{M}$. The 3T3-L1 cells were differentiated into adipocytes by day 8 . 


\section{The ROC and enrichment plots of the Bayesian classification model}
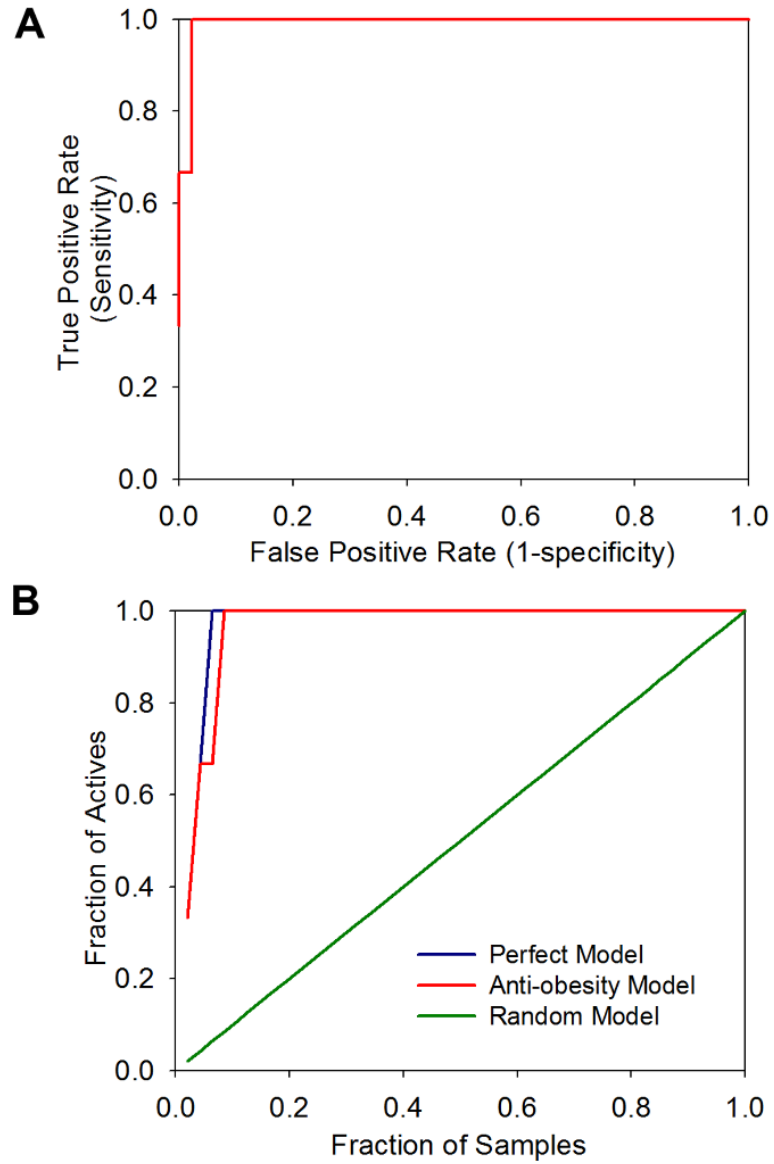

Figure S1. ROC and enrichment plots of the Bayesian classification model for the validation set. (A) ROC plot. (B) Enrichment plot of anti-obesity model (red), overlap with those of the perfect (blue) and random model (green). 


\section{Immunofluorescence Analysis of Compound 6}

The 3T3-L1 cells grown in a 8-well culture slide and treated with compound $\mathbf{6}$, and then The cells were incubated with $500 \mu \mathrm{L}$ of methanol/acetone $=1 / 1$ mixture for $5 \mathrm{~min}$. After washing with PBS four times, the cells were incubated with PBSTr solution for $5 \mathrm{~min}$, and then blocked with 5\% skim milk. The cells were made to react with PPAR- $\gamma$ primary antibody followed by anti-rabbit secondary antibody and was strained with DAPI (4',6-diamidino-2phenylindole). DAPI nuclear staining was observed using a fluorescent microscope.

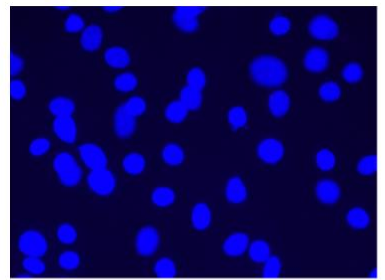

Control

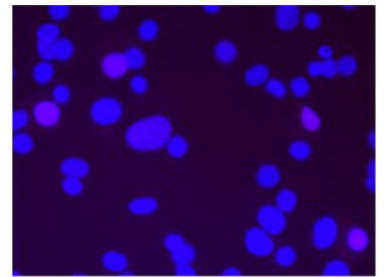

Induction

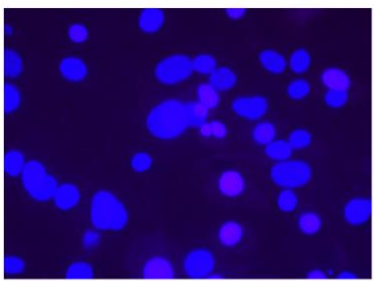

Induction + Compound $6(1 \mu \mathrm{M})$

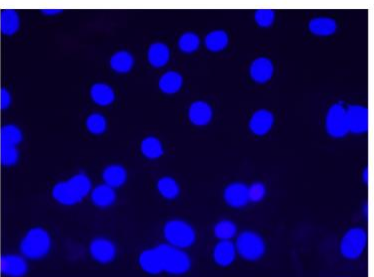

Induction + Compound $6(5 \mu \mathrm{M})$

Figure S2. Immunofluorescence analysis for PPAR- $\gamma$ in 3T3-L1 cells. Red color indicated PPAR- $\gamma$. 


\section{Data for the 50 Virtual Hit Compounds}

Table S2. Computational and Biological Data for the 50 Virtual Hit Compounds

\begin{tabular}{|c|c|c|c|c|}
\hline \multirow{2}{*}{ Compound ID } & \multicolumn{2}{|c|}{ Virtual Screening Results } & \multirow{2}{*}{$\begin{array}{l}\text { Lipid inhibitory } \\
\text { activity }(\%)^{c}\end{array}$} & \multirow{2}{*}{$\begin{array}{c}\text { Cell viability } \\
(\%)^{d}\end{array}$} \\
\hline & EON_Rank ${ }^{a}$ & EON_ET_combo ${ }^{b}$ & & \\
\hline VS_01 & 1 & 1.778 & 12.3 & 83.6 \\
\hline $\begin{array}{c}\text { VS_02 } \\
\text { (compound 1) }\end{array}$ & 2 & 1.728 & 20.1 & 107.8 \\
\hline VS_03 & 3 & 1.723 & -0.1 & 94.6 \\
\hline VS_04 & 4 & 1.722 & 18.9 & 98.3 \\
\hline VS_05 & 5 & 1.708 & -7.5 & 71.2 \\
\hline VS_06 & 6 & 1.705 & 11.5 & 93.8 \\
\hline VS_07 & 7 & 1.698 & -43 & 88.6 \\
\hline VS_08 & 8 & 1.686 & -23.5 & 117.2 \\
\hline VS_09 & 9 & 1.683 & -37.3 & 95.5 \\
\hline VS_10 & 10 & 1.670 & 27.1 & 92.0 \\
\hline VS_11 & 11 & 1.657 & 2.7 & 105.8 \\
\hline VS_12 & 12 & 1.656 & -2.2 & 79.5 \\
\hline VS_13 & 13 & 1.649 & -7.4 & 111.0 \\
\hline VS_14 & 14 & 1.646 & 74.9 & 59.9 \\
\hline VS_15 & 15 & 1.639 & 1.6 & 114.5 \\
\hline VS_16 & 16 & 1.639 & -24.3 & 111.4 \\
\hline VS_17 & 17 & 1.636 & 19.5 & 85.8 \\
\hline VS_18 & 18 & 1.618 & 8 & 83.9 \\
\hline VS_19 & 19 & 1.615 & -30.2 & 102.2 \\
\hline VS_20 & 20 & 1.614 & -6.6 & 78.6 \\
\hline VS_21 & 21 & 1.612 & 11.1 & 96.0 \\
\hline VS_22 & 22 & 1.603 & -0.5 & 97.1 \\
\hline VS_23 & 23 & 1.598 & -11.3 & 114.8 \\
\hline $\begin{array}{c}\text { VS_24 } \\
\text { (compound 6) }\end{array}$ & 24 & 1.598 & 102.2 & 93.1 \\
\hline VS_25 & 25 & 1.592 & 9.8 & 110.0 \\
\hline $\begin{array}{c}\text { VS_26 } \\
\text { (compound 4) }\end{array}$ & 26 & 1.591 & 20.6 & 107.6 \\
\hline VS_27 & 27 & 1.589 & 23.4 & 89.0 \\
\hline VS_28 & 28 & 1.587 & -12.7 & 106.2 \\
\hline VS_29 & 29 & 1.576 & -27.6 & 101.1 \\
\hline $\begin{array}{c}\text { VS_30 } \\
\text { (compound 2) }\end{array}$ & 30 & 1.574 & 59.1 & 106.4 \\
\hline VS_31 & 31 & 1.568 & 7.6 & 93.5 \\
\hline VS_32 & 32 & 1.556 & -10.1 & 96.6 \\
\hline VS_33 & 33 & 1.552 & 29.1 & 73.7 \\
\hline
\end{tabular}




$\begin{array}{ccccc}\text { VS_34 } & 34 & 1.547 & 37.0 & 106.4 \\ \text { (compound 5) } & 35 & 1.533 & 17.0 & 75.1 \\ \text { VS_35 } & 36 & 1.530 & -12.9 & 111.2 \\ \text { VS_36 } & 37 & 1.528 & 19.0 & 74.1 \\ \text { VS_37 } & 38 & 1.517 & 14.9 & 95.4 \\ \text { VS_38 } & 39 & 1.517 & -27.3 & 73.5 \\ \text { VS_39 } & 40 & 1.514 & -45.8 & 105.5 \\ \text { VS_40 } & 41 & 1.477 & 0.8 & 99.5 \\ \text { VS_41 } & 42 & 1.462 & -41.1 & 112.8 \\ \text { VS_42 } & 43 & 1.460 & 10.2 & 78.0 \\ \text { VS_43 } & 44 & 1.455 & 57.3 & 100.9 \\ \text { VS_44 } & 45 & 1.444 & 18.9 & 81.8 \\ \text { (compound 3) } & 46 & 1.443 & -4.2 & 81.0 \\ \text { VS_45 } & 47 & 1.429 & -80.9 & 97.7 \\ \text { VS_46 } & 48 & 1.428 & 8 & 90.2 \\ \text { VS_47 } & 49 & 1.427 & 24.8 & 82.7 \\ \text { VS_48 } & 50 & 1.405 & -20.2 & 95.0 \\ \text { VS_49 } & \text { VS_50 } & 45 & \end{array}$

${ }^{a}$ Rank of the 50 virtual hit compounds according to the EON_ET_combo scores. ${ }^{b}$ Sum of the Shape Tanimoto (ST) and the Electrostatic Tanimoto (ET_pb). ${ }^{c}$ The selected 50 compounds were tested on 3T3-L1 cells at $5 \mu \mathrm{M}$. The 3T3-L1 cells were differentiated into adipocytes by day $8 .{ }^{d}$ Cell viability effects of 50 compounds were tested on $3 \mathrm{~T} 3-\mathrm{L} 1$ cells at $5 \mu \mathrm{M}$. 


\section{Purity Analysis of NED-240 and Compounds 1-6}

NED-240

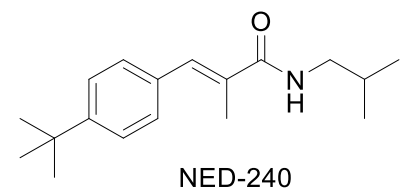

Scheme S1. Chemical structure of NED-240

Purity: 99\%

The HPLC analysis was performed using a reversed-phase Agilent Eclipse Plus C18 column $(4.6 \times 100 \mathrm{~mm}, 3.5 \mu \mathrm{m})$ at a flow rate of $0.7 \mathrm{~mL} / \mathrm{min}(30-100 \%$ aqueous $\mathrm{MeOH}$ with $0.1 \%$ formic acid over $20 \mathrm{~min}$ and $\mathrm{MeOH}$ with $0.1 \%$ formic acid from 20 to $25 \mathrm{~min}$ ).

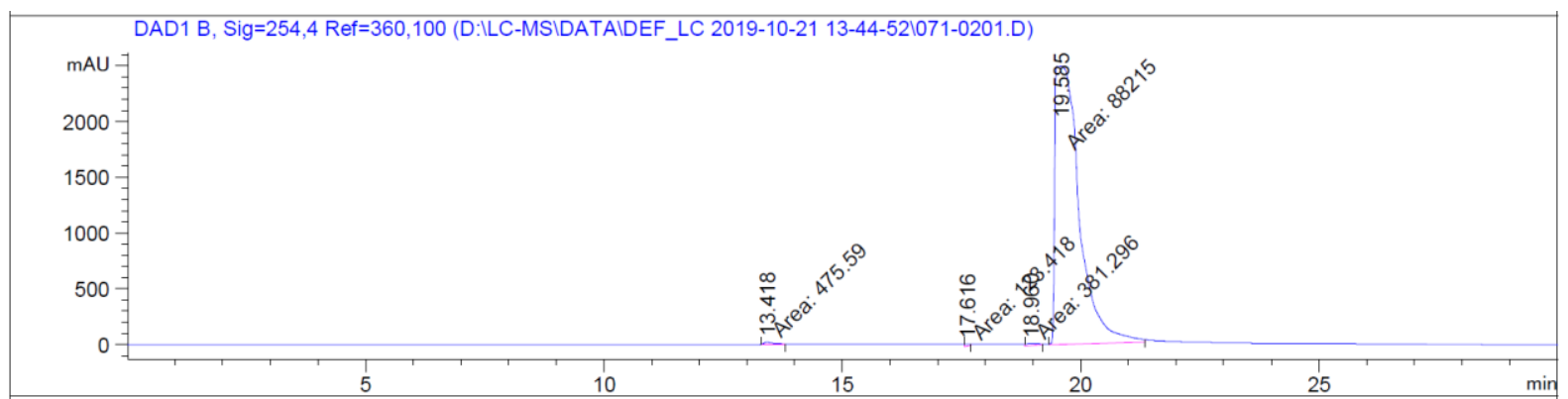

\begin{tabular}{|c|c|c|c|c|c|c|}
\hline$\#$ & Time & Area & Height & Width & Area $\%$ & Symmetry \\
\hline 1 & 13.418 & 475.6 & 25.5 & 0.3104 & 0.533 & 0.331 \\
\hline 2 & 17.616 & 123.4 & 18 & 0.1055 & 0.138 & 0.575 \\
\hline 3 & 18.96 & 381.3 & 20.8 & 0.3051 & 0.427 & 0.678 \\
\hline 4 & 19.585 & 88215 & 2493.8 & 0.5896 & 98.901 & 0.306 \\
\hline
\end{tabular}

Figure S3. HPLC purity data of NED-240 


\section{Compound 1}<smiles>Cc1ccc(OCC(=O)Nc2ccc3c(c2)OCO3)c([N+](=O)[O-])c1</smiles>

Scheme S2. Chemical structure of compound 1

Purity: $100 \%$

The LC/MS analysis was performed using a reversed-phase BEH C18 column $(2.1 \times 50 \mathrm{~mm}$, $1.7 \mu \mathrm{m})$ at a flow rate of $0.4 \mathrm{~mL} / \mathrm{min}(10-90 \%$ aqueous $\mathrm{MeCN}$ with $0.2 \%$ TFA over $2.8 \mathrm{~min}$ and $90-10 \%$ aqueous $\mathrm{MeCN}$ with $0.2 \%$ TFA from 2.8 to $5 \mathrm{~min}$ ).

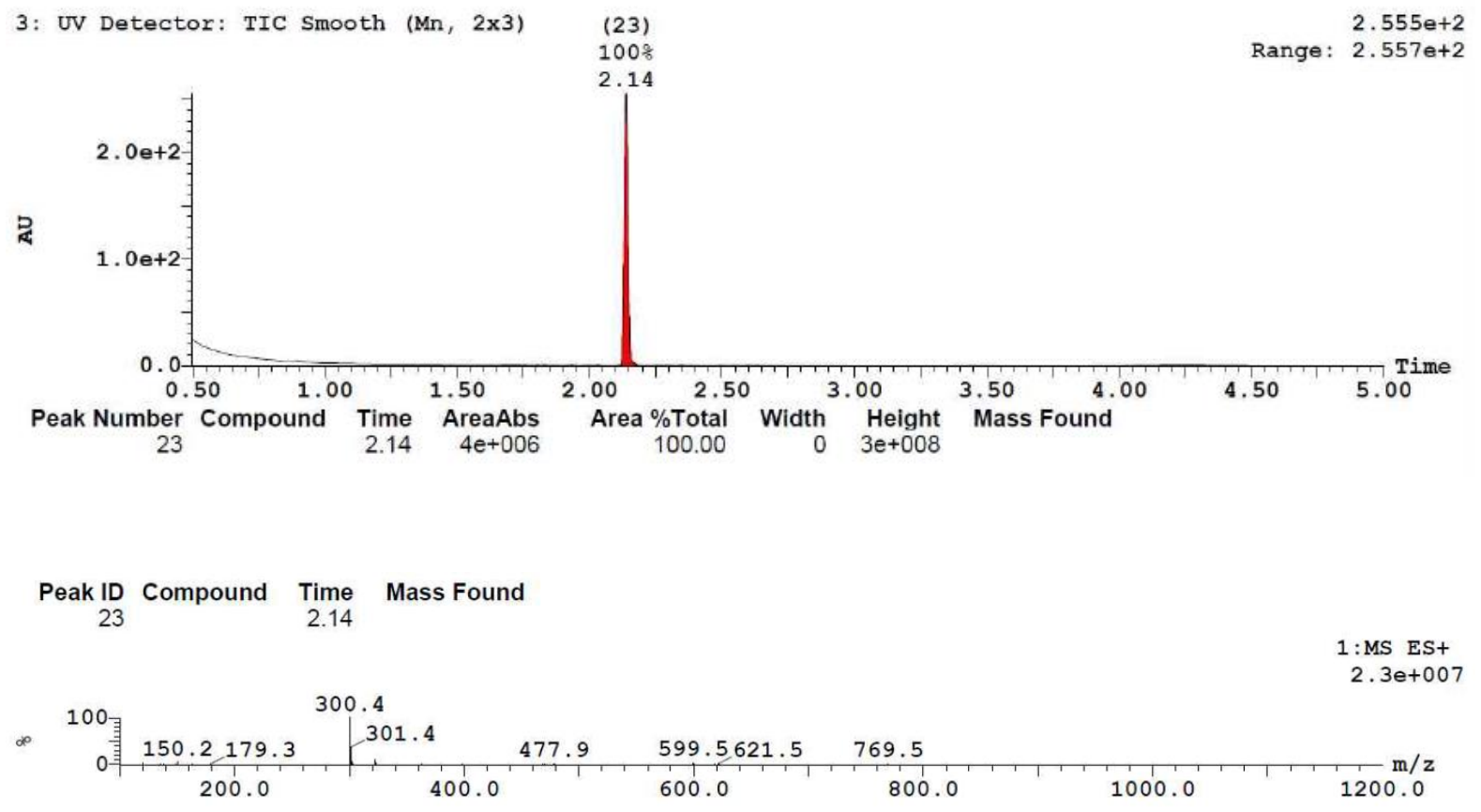

Figure S4. LC/MS purity analysis of compound 1 


\section{Compound 2}

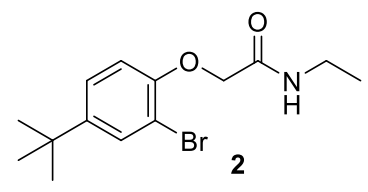

Scheme S3. Chemical structure of compound 2

Purity: $100 \%$

The LC/MS analysis was performed using a reversed-phase BEH C18 column $(2.1 \times 50 \mathrm{~mm}$, $1.7 \mu \mathrm{m})$ at a flow rate of $0.4 \mathrm{~mL} / \mathrm{min}(10-90 \%$ aqueous $\mathrm{MeCN}$ with $0.2 \%$ TFA over $2.8 \mathrm{~min}$ and $90-10 \%$ aqueous $\mathrm{MeCN}$ with $0.2 \%$ TFA from 2.8 to $5 \mathrm{~min}$ ).

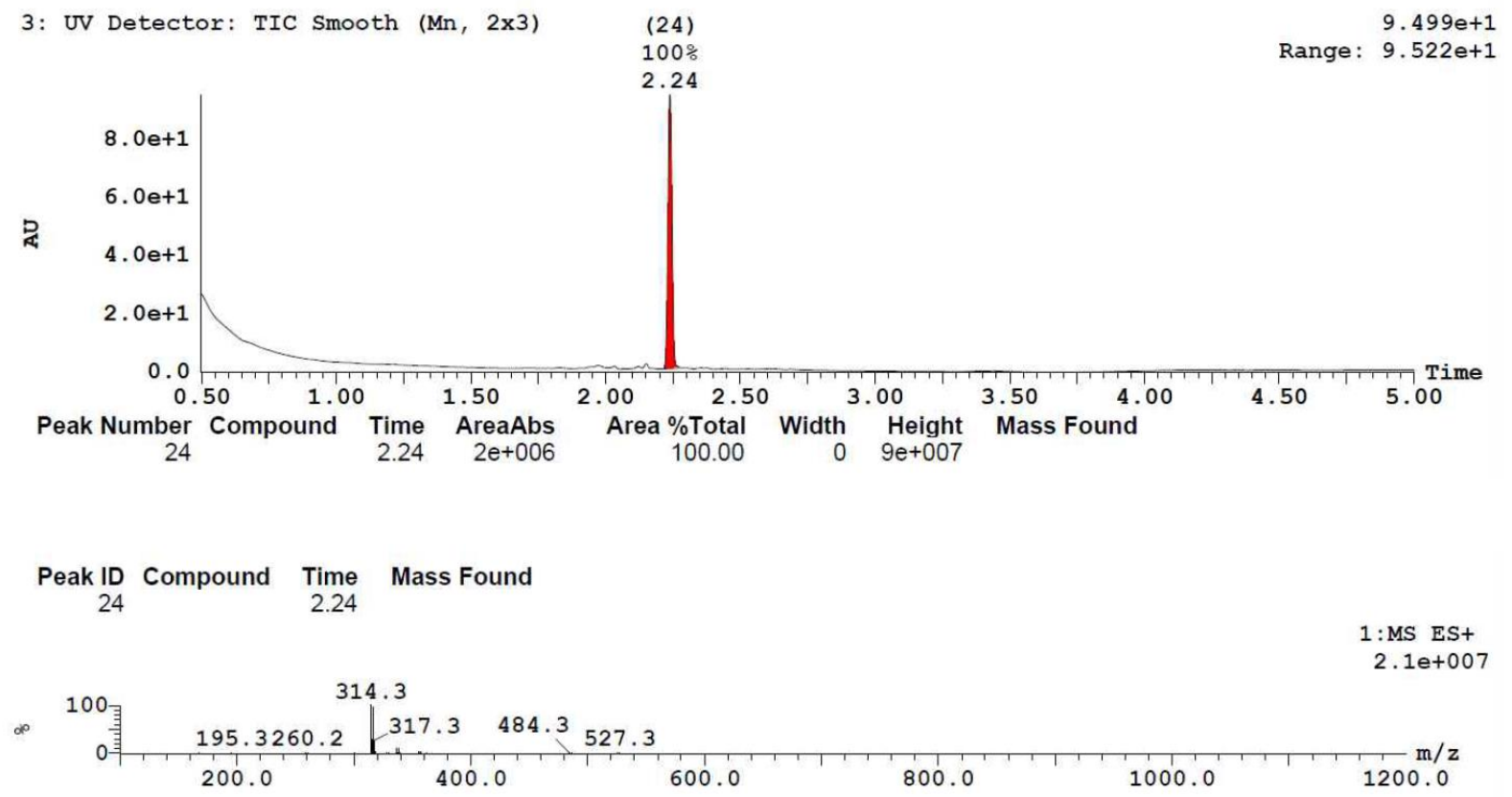

Figure S5. LC/MS purity analysis of compound 2 


\section{Compound 3}<smiles>Cc1nnc(SCC(=O)NCCOc2ccccc2)s1</smiles>

Scheme S4. Chemical structure of compound 3

Purity: 100\%

The LC/MS analysis was performed using a reversed-phase BEH C18 column $(2.1 \times 50 \mathrm{~mm}$, $1.7 \mu \mathrm{m})$ at a flow rate of $0.4 \mathrm{~mL} / \mathrm{min}(10-90 \%$ aqueous $\mathrm{MeCN}$ with $0.2 \%$ TFA over $2.8 \mathrm{~min}$ and $90-10 \%$ aqueous $\mathrm{MeCN}$ with $0.2 \%$ TFA from 2.8 to $5 \mathrm{~min}$ ).

3: UV Detector: TIC Smooth (Mn, 2x3)

1.23e+2

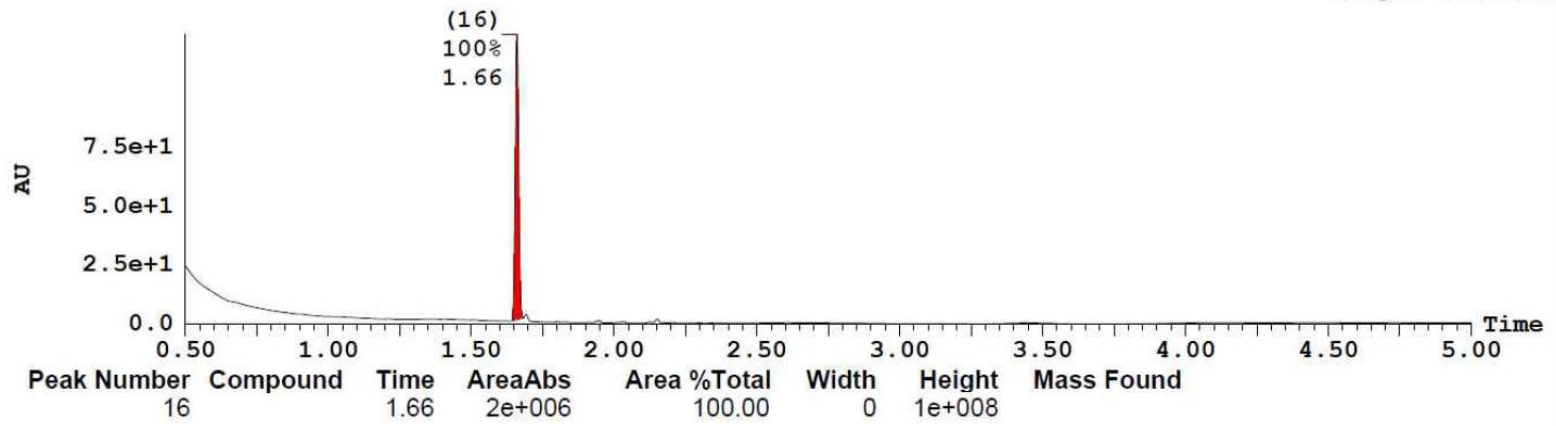

Peak ID Compound Time
16 $\quad$ Mass Found

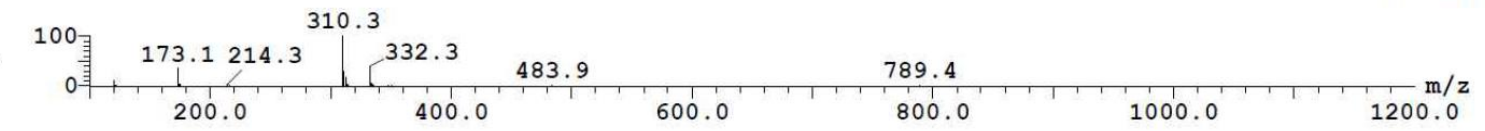

Figure S6. LC/MS purity analysis of compound 3 


\section{Compound 4}

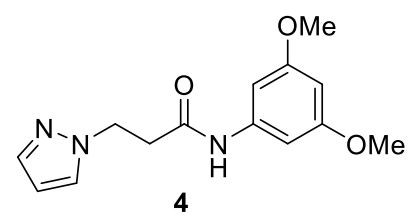

Scheme S5. Chemical structure of compound 4

Purity: $100 \%$

The LC/MS analysis was performed using a reversed-phase BEH C18 column $(2.1 \times 50 \mathrm{~mm}$, $1.7 \mu \mathrm{m})$ at a flow rate of $0.4 \mathrm{~mL} / \mathrm{min}(10-90 \%$ aqueous $\mathrm{MeCN}$ with $0.2 \%$ TFA over $2.8 \mathrm{~min}$ and $90-10 \%$ aqueous $\mathrm{MeCN}$ with $0.2 \%$ TFA from 2.8 to $5 \mathrm{~min}$ ).

3: UV Detector: TIC Smooth (Mn, 2x3)
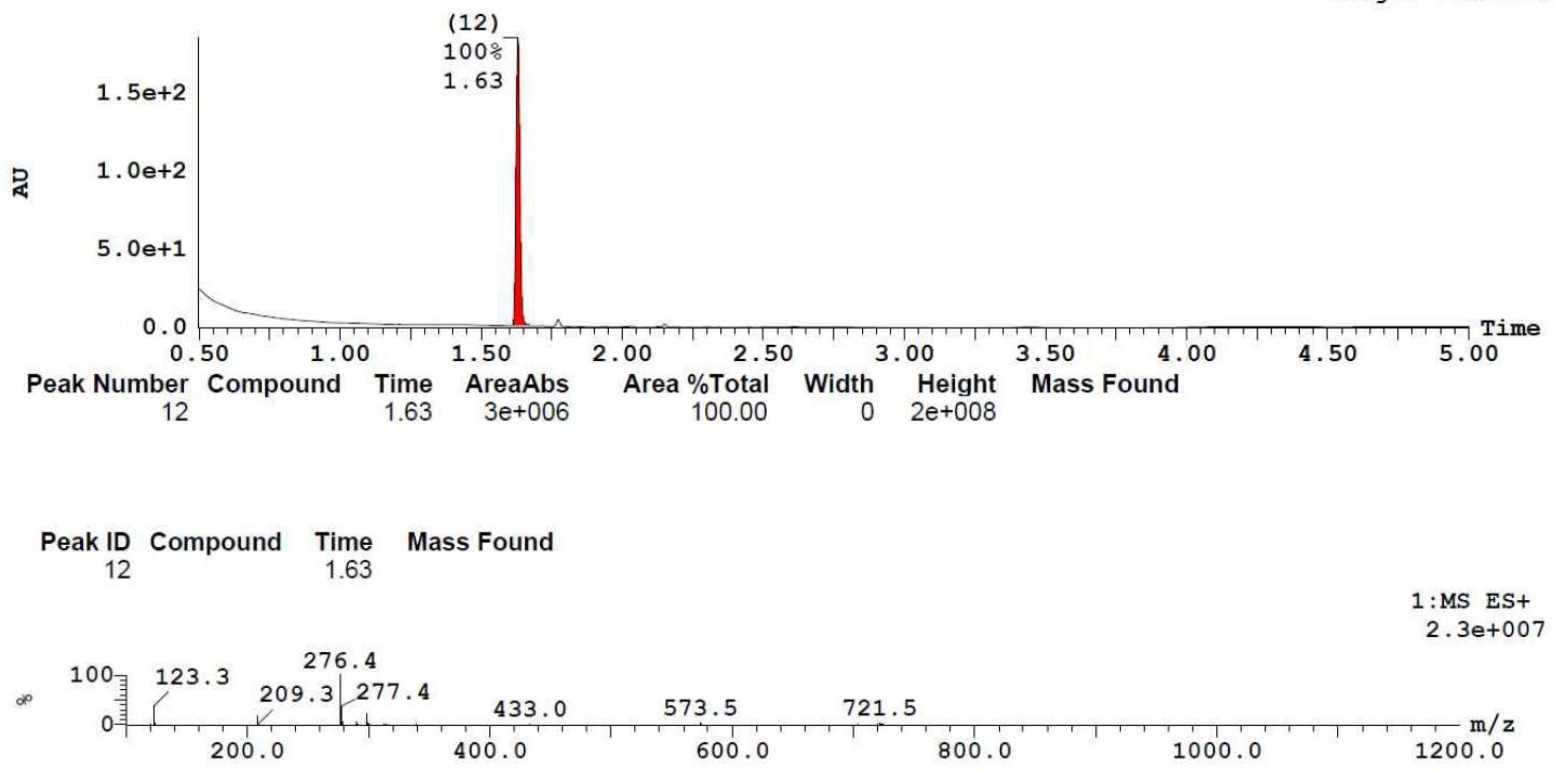

Figure S7. LC/MS purity analysis of compound 4 


\section{Compound 5}

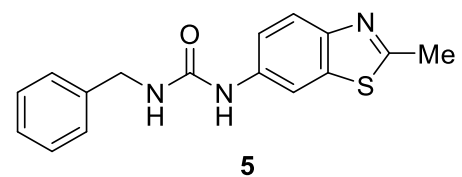

Scheme S6. Chemical structure of compound 5

Purity: $100 \%$

The LC/MS analysis was performed using a reversed-phase BEH C18 column $(2.1 \times 50 \mathrm{~mm}$, $1.7 \mu \mathrm{m})$ at a flow rate of $0.4 \mathrm{~mL} / \mathrm{min}(10-90 \%$ aqueous $\mathrm{MeCN}$ with $0.2 \%$ TFA over $2.8 \mathrm{~min}$ and $90-10 \%$ aqueous $\mathrm{MeCN}$ with $0.2 \%$ TFA from 2.8 to $5 \mathrm{~min}$ ).

3: UV Detector: TIC Smooth (Mn, 2x3)

$2.849 e+2$

Range: $2.852 e+2$
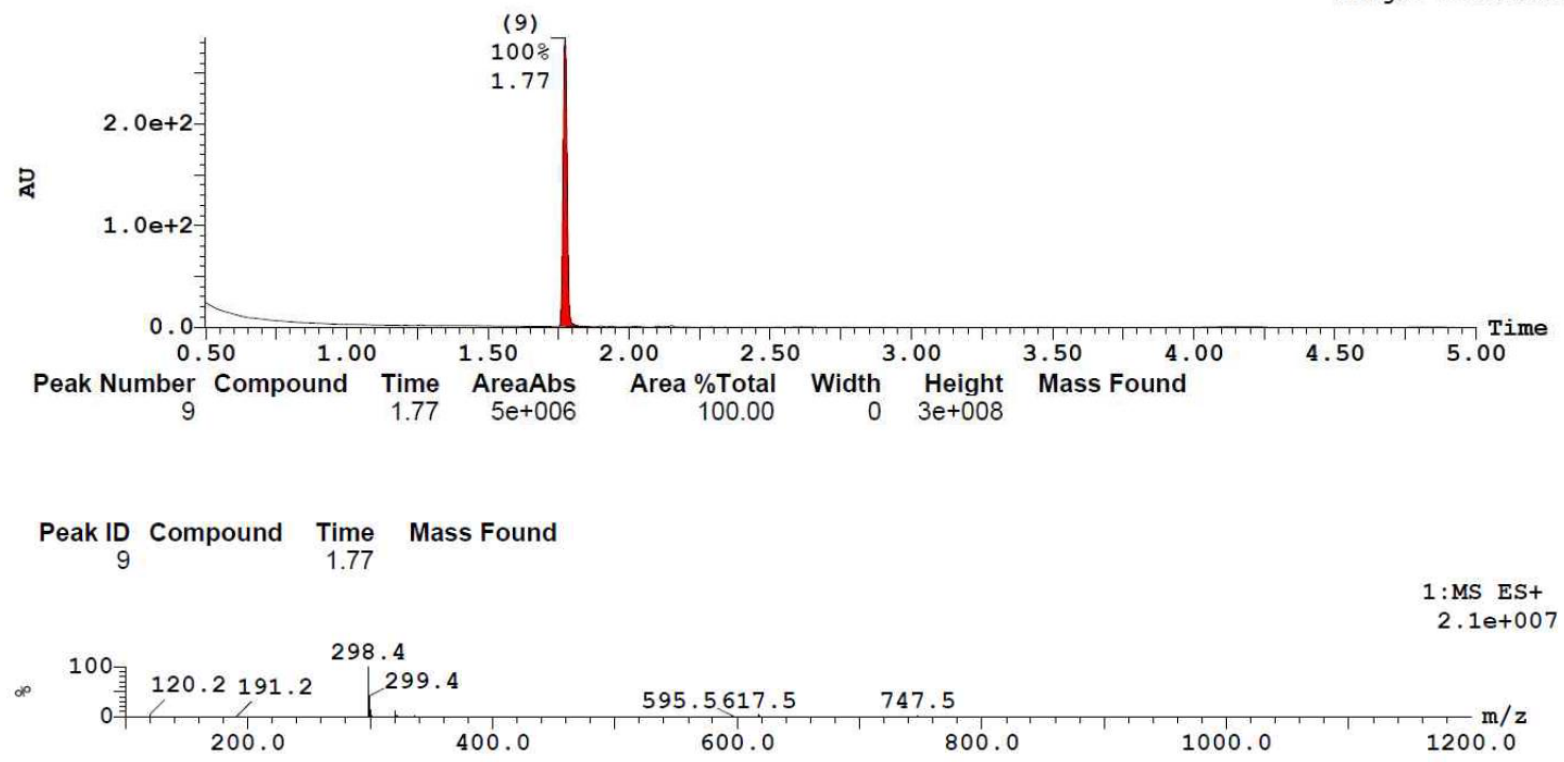

Figure S8. LC/MS purity analysis of compound 5 


\section{Compound 6}

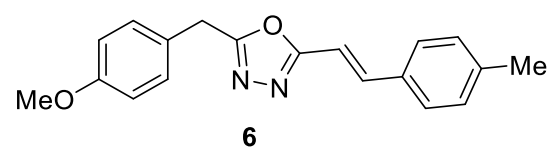

Scheme S7. Chemical structure of compound 6

Purity: 99\%

The HPLC analysis was performed using a reversed-phase Agilent Eclipse Plus C18 column $(4.6 \times 100 \mathrm{~mm}, 3.5 \mu \mathrm{m})$ at a flow rate of $0.7 \mathrm{~mL} / \mathrm{min}(30-100 \%$ aqueous $\mathrm{MeOH}$ with $0.1 \%$ formic acid over $20 \mathrm{~min}$ and $\mathrm{MeOH}$ with $0.1 \%$ formic acid from 20 to $25 \mathrm{~min}$ ).

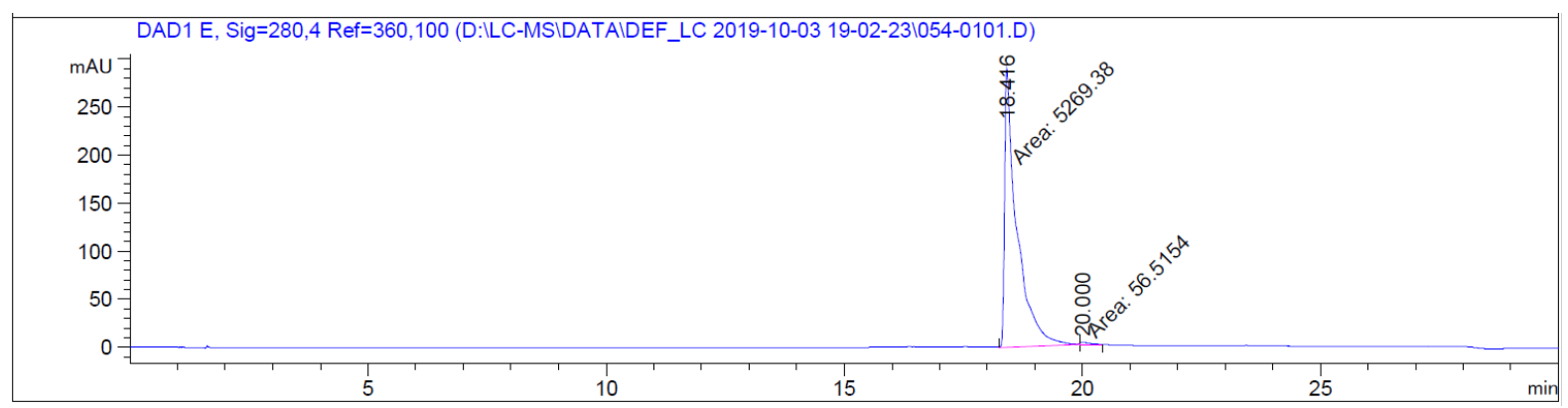

\begin{tabular}{|c|c|c|c|c|c|c|} 
\# & \multicolumn{1}{c}{ Time } & \multicolumn{1}{c}{ Area } & Height & \multicolumn{1}{c}{ Width } & \multicolumn{1}{c}{ Area\% } & Symmetry \\
\hline 1 & 18.416 & 5269.4 & 290.9 & 0.3019 & 98.939 & 0.226 \\
\hline 2 & 20 & 56.5 & 3.3 & 0.2898 & 1.061 & 0.126 \\
\hline
\end{tabular}

Figure S9. LC/MS purity analysis of compound 6 


\section{NMR Copies of Compound 6}

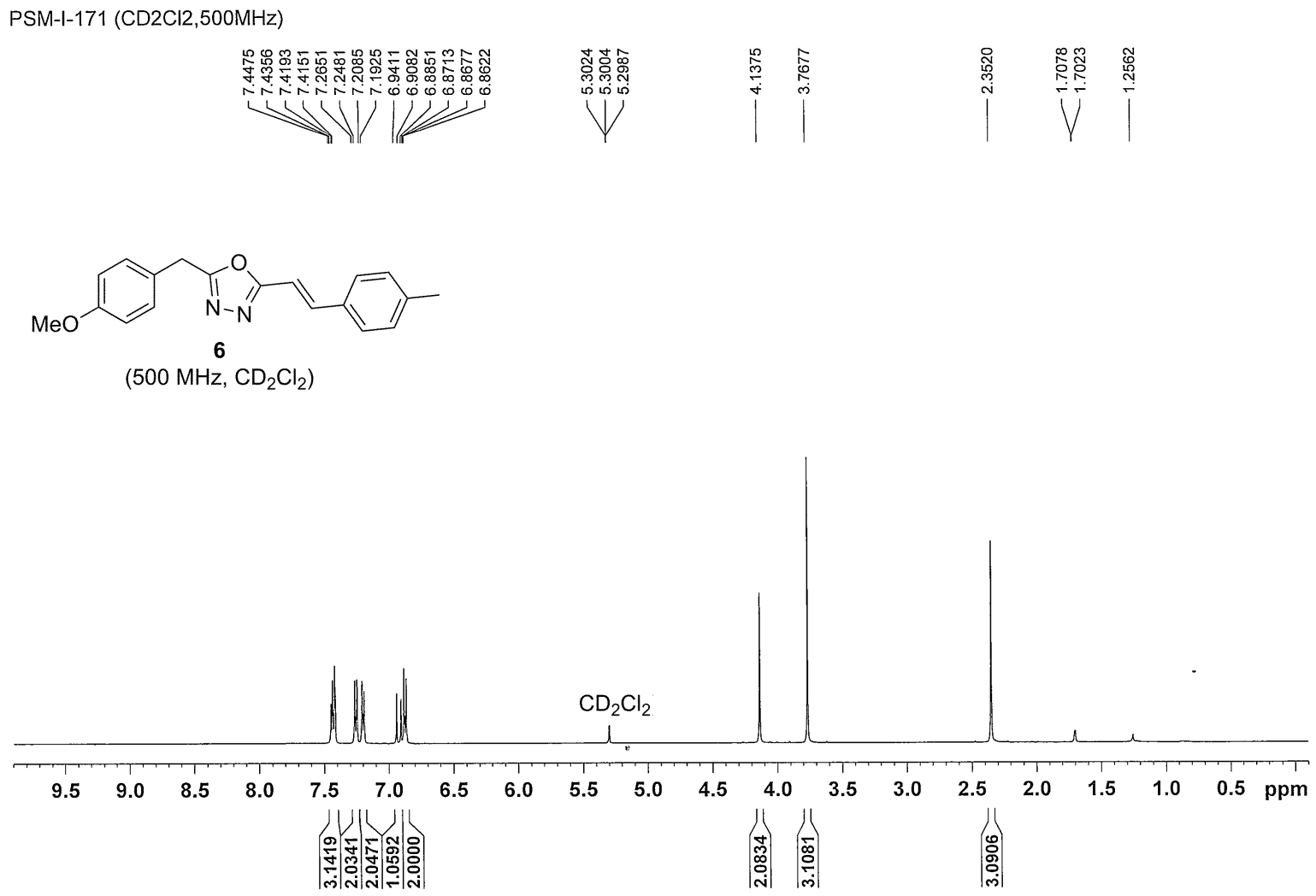

Figure S10. ${ }^{1} \mathrm{H}$ NMR spectra of compound 6

PSM-I-171 (CD2Cl2,500MHz)

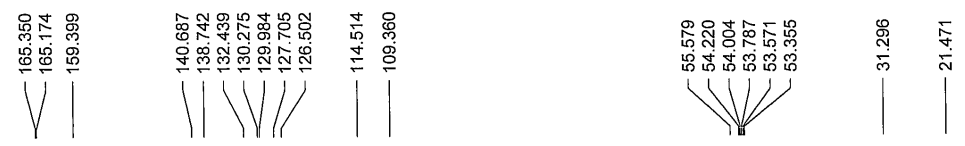

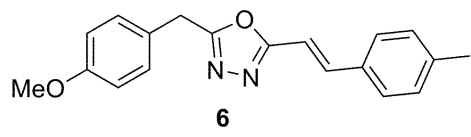

(125 MHz, $\mathrm{CD}_{2} \mathrm{Cl}_{2}$ )

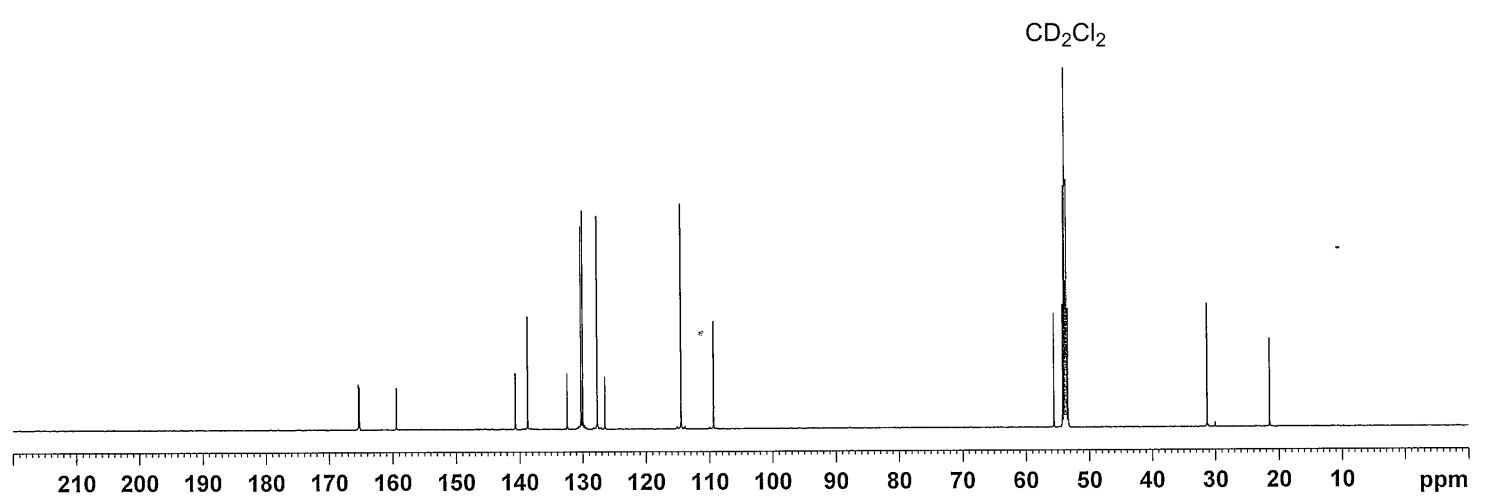

Figure S11. ${ }^{13} \mathrm{C}$ NMR spectra of compound 6 